\title{
Mise au point de techniques analytiques pour la spéciation du sélénium dans les boues de stations d'épuration d'eaux résiduaires urbaines
}

\section{Development of new analytical techniques for determining selenium speciation in sewage sludge: differential pulse cathodic stripping voltammetry (DPCSV) and electrothermal atomic adsorption spectrometry (ETAAS)}

\author{
I. Heninger, M. Potin-Gautier, M. Astruc, L. Galvez et V. Vignier
}

Volume 11, numéro 3, 1998

URI : https://id.erudit.org/iderudit/705310ar

DOI : https://doi.org/10.7202/705310ar

\section{Aller au sommaire du numéro}

\section{Éditeur(s)}

Université du Québec - INRS-Eau, Terre et Environnement (INRS-ETE)

\section{ISSN}

0992-7158 (imprimé)

1718-8598 (numérique)

\section{Découvrir la revue}

Citer cet article

Heninger, I., Potin-Gautier, M., Astruc, M., Galvez, L. \& Vignier, V. (1998). Mise au point de techniques analytiques pour la spéciation du sélénium dans les boues de stations d'épuration d'eaux résiduaires urbaines. Revue des sciences de l'eau / Journal of Water Science, 11(3), 333-346.

https://doi.org/10.7202/705310ar

\section{Résumé de l'article}

Les stations d'épuration d'eaux résiduaires sont une des étapes du cycle du sélénium dans l'environnement et contribuent à sa redistribution dans le milieu naturel. Très peu étudié jusqu'à présent dans ces milieux, le sélénium n'en est pas moins un élément très important du point de vue écotoxicologique, sa teneur dans les boues de stations d'épuration destinées à l'épandage agricole faisant par ailleurs l'objet d'une norme.

Nous avons mis au point des techniques permettant la détermination spécifique de l'élément total dans ce type d'échantillon, par minéralisation classique ou assistée par micro-ondes et dosage par Voltamétrie de Redissolution Cathodique Différentielle Pulsée (DPCSV) et Spectrométrie d'Absorption Atomique ElectroThermique (ETAAS). Le contrôle qualité a été effectué sur deux échantillons certifiés fournis par le Bureau Communautaire de Référence (BCR) : la boue CRM 145 R et la boue CRM 007.

Cependant, lorsqu'on parle de risque toxicologique, il est important de s'intéresser à la détermination des différentes formes sous lesquelles cet élément peut être présent. Nous avons pour cela réalisé des extractions parallèles (spéciation de phases) du sélénium contenu dans les boues afin de déterminer quel pourcentage du sélénium total est réellement et potentiellement disponible pour les végétaux lors d'un épandage sur sol agricole. La spéciation d'espèces a été brièvement abordée dans le but de déterminer les teneurs en Se(IV) et Se(VI), espèces les plus toxiques. 


\section{Mise au point de techniques analytiques pour la spéciation du sélénium dans les boues de stations d'épuration d'eaux résiduaires urbaines}

\section{Development of new analytical techniques for determining selenium speciation in sewage sludge: differential pulse cathodic stripping voltammetry (DPCSV) and electro- thermal atomic absorption spectrometry (ETAAS)}

\section{HENINGER ${ }^{1}$, M. POTIN-GAUTIER ${ }^{1}$ *, M. ASTRUC ${ }^{1}$, L. GALVEZ ${ }^{2}$, V. VIGNIER ${ }^{2}$}

Reçu le 12 mai 1997, accepté le 20 janvier $1998^{\star *}$.

\section{SUMMARY}

The great effort undertaken for about twenty years to improve the quality of surface waters has led to the construction of numerous waste treatments plants, generating an increasing amount of sludge. Waste water and sludge treatment processes represent an important point in the hydrological cycle at which the disposal of substantial quantities of trace elements to the environment may be regulated. From the law on waste recovery and disposal in 1975 to the European guideline about wastes in 1991, the priority has been given to waste recovery and recycling. With increasing pressure to ban all sludge dumping at sea, and considering the prohibitive costs of land-filling and incineration, there is a great tendency to dispose of sludge on land $(40 \%$ in 1988 to $60 \%$ in 1992).

Although numerous studies have demonstrated the intrinsic value of sludge for soil amendment, given its nitrogen, phosphorus and homogeneous organic matter content, evidence has accumulated in recent years that numerous environmental problems can arise because of the presence in sludges of high amounts of certain trace elements (potentially toxic to plants and to human beings and liable to be concentrated along the food chain), among which selenium is particularly interesting.

Selenium presents a complex case, as it is also an essential element for living organisms (including humans). The amendment with sewage sludge is sometismes used to increase the selenium content in crops, and afterwards in cattle, when there is a proven lack of this element in a given place. Nevertheless the boundary between essentiality and toxicity is relatively narrow and is expressed at trace levels. It is thus particularly important to survey the sele-

1. Laboratoire de Chimie Analytique, Université de Pau et des Pays de l'Adour, 64000 Pau, France.

2. CIRSEE, 38, rue du Président Wilson, 78230 Le Pecq, France.

Communication présentée au Colloque International du GRUTTEE, " Les sous-produits de traitement et d'épuration des eaux ", mars 1997 à Rennes.

* Correspondance.

* * Les commentaires seront reçus jusqu'au 30 avril 1999. 
nium concentrations encountered in sewage sludge, especially as guidelines and regulations concerning these data will probably be strengthened.

Presently, in France, sludge must not contain more than $200 \mathrm{mgSe} \cdot \mathrm{kg}^{-1}$ dry weight and must not be used on soils containing more than $10 \mathrm{mg} \mathrm{Se} \cdot \mathrm{kg}^{-1}$ dry weight (AFNOR U 44-041 norm). This norm concerns only the total amount of selenium contained in sludge and does not take into account the different species (organic and inorganic $\mathrm{Se}(-\mathrm{II}), \mathrm{Se}(0), \mathrm{Se}(\mathrm{IV})$ and $\mathrm{Se}(\mathrm{VI}))$ that could be present.

First of all we had to develop methods for the classical and microwave-assisted wet digestion of sewage sludge, and the determination of their total selenium concentration by Differential Pulse Cathodic Stripping Voltammetry (DPCSV) and ElectroThermal Atomic Absorption Spectrometry (ETAAS). Quality assurance involved the analysis of two BCR (Community Bureau of Reference) certified sewage sludge reference materials (CRM $145 \mathrm{R}$ and CRM 007) and the different techniques were then applied to natural samples from a representative French sewage treatment plant located in the city of Tarbes (South-West of France).

The mixture $\mathrm{HNO}_{3}-\mathrm{H}_{2} \mathrm{O}_{2}-\mathrm{H}_{2} \mathrm{O}$ led to the best results for the digestion and analysis of certified samples, caused few problems for the analysis by DPCSV and ETAAS, and was therefore retained. The decrease of the digestion duration obtained by the use of microwaves was particularly interesting (from one to three days on a hot plate to less than one hour by the Microdigest 301 (PROLABO, France)), and reproducibility was also acceptable (between 3 and $10 \%$ ). Concentrations obtained for the sewage sludge from the Tarbes treatment plant were very much lower than those for NF U 44-041: $1.08 \pm 0.11 \mathrm{mg}$ $\mathrm{Se} \cdot \mathrm{kg}^{-1}$ dry weight.

However knowledge of speciation, that is to say the determination of the different physicochemical forms of selenium present in a given medium, is necessary when speaking of the toxicological risk represented by an element. The mobility of selenium and its toxicity to the biosphere are related to its association with various sludge or soil constituents as well as to its total concentration. "Soft" or partial extraction techniques are necessary when the aim of the study to determine trace element speciation. The extractants use must separate selenium from the matrix without inducing any loss or change in the partitioning of individual chemical species. In parallel extractions the mechanisms involved for each extractant must correspond to processes occurring in nature and are then associated with special fractions of selenium: soluble, exchangeable, "oxidizable", and "mineral" fractions.

Parallel extractions with three types of extractants were chose for this study and applied first to CRM 007: warm water (soluble fraction), ammonium phosphate-citric acid (soluble + exchangeable fraction) and sodium hydroxide (soluble + exchangeable + "oxidizable" fraction). The soluble, exchangeable, "oxidizable" and "mineral" fractions represent respectively: 11\%, 14\%, 39\% and $36 \%$. The same procedure was then applied to natural samples from Tarbes giving the following results: $36 \%$ soluble, $22 \%$ exchangeable, $42 \%$ "oxidizable". The sodium hydroxide extraction procedure allowed us to extract the entire Se content of this sludge $\left(1.07 \pm 0.03 \mathrm{mg} \mathrm{Se} \cdot \mathrm{kg}^{-1}\right.$ dry weight), showing that all the selenium present is potentially available after agricultural land application. It was then possible in this fraction to deal with the species speciation of selenium by the mean of a separation of inorganic and inorganic species on an Amberlite CG-400 resin and a specific analysis by DPCSV. Se(IV) and Se(VI) represent respectively between 30 and $40 \%$ and between 2 and $20 \%$ of total selenium in the sludges from Tarbes. 


\section{RÉSUMÉ}

Les stations d'épuration d'eaux résiduaires sont une des étapes du cycle du sélénium dans l'environnement et contribuent à sa redistribution dans le milieu naturel. Très peu étudié jusqu'à présent dans ces milieux, le sélénium n'en est pas moins un élément très important du point de vue écotoxicologique, sa teneur dans les boues de stations d'épuration destinées à l'épandage agricole faisant par ailleurs l'objet d'une norme.

Nous avons mis au point des techniques permettant la détermination spécifique de l'élément total dans ce type d'échantillon, par minéralisation classique ou assistée par micro-ondes et dosage par Voltamétrie de Redissolution Cathodique Différentielle Pulsée (DPCSV) et Spectrométrie d'Absorption Atomique ElectroThermique (ETAAS). Le contrôle qualité a été effectué sur deux échantillons certifiés fournis par le Bureau Communautaire de Référence (BCR) : la boue CRM 145 R et la boue CRM 007.

Cependant, lorsqu'on parle de risque toxicologique, il est important de s'intéresser à la détermination des différentes formes sous lesquelles cet élément peut être présent. Nous avons pour cela réalisé des extractions parallèles (spéciation de phases) du sélénium contenu dans les boues afin de déterminer quel pourcentage du sélénium total est réellement et potentiellement disponible pour les végétaux lors d'un épandage sur sol agricole. La spéciation d'espèces a été brièvement abordée dans le but de déterminer les teneurs en Se(IV) et $\mathrm{Se}(\mathrm{VI})$, espèces les plus toxiques.

Mots clés : sélénium, boues de station d'épuration, spéciation, minéralisation, extraction.

\section{INTRODUCTION}

Les traitements réservés aux eaux résiduaires et aux boues de stations d'épuration représentent un stade important du cycle hydrobiologique, au cours duquel la redistribution de quantités substantielles de métaux dans l'environnement doit être régulée.

Depuis la loi cadre française du 15 juillet 1975 jusqu'à la directive cadre européenne reprise dans la circulaire du 8 janvier 1991, la priorité est donnée à la récupération et au recyclage des déchets (VALIRON, 1994). La valorisation agricole des boues en constitue la forme la plus répandue à l'heure actuelle en Europe (60\% en 1992) (PERRIN et IMPENS, 1995).

Bien que de nombreuses études aient mis en évidence l'intérêt des boues pour l'amendement des sols agricoles par l'apport d'azote, de phosphore et de matière organique (STERRITT et LESTER, 1980), il a également été clairement montré ces dernières années que de sérieux problèmes environnementaux peuvent provenir du fait que ces boues sont susceptibles de contenir de fortes teneurs en éléments traces (CAMBIER, 1994). Ces éléments sont pour la plupart potentiellement toxiques pour les plantes et les êtres vivants et susceptibles de se concentrer le long de la chaîne alimentaire. Le cas du sélénium est complexe dans la mesure où il est à la fois un oligo-élément, indispensable à la vie, et un toxique, et ce dans un domaine étroit de concentration (DUBOIS et BELLEVILLE, 1988). Son devenir dans les stations d'épuration est encore très mal connu, faute 
d'études approfondies et malgré l'existence d'une norme sur sa teneur dans les boues destinées à l'épandage: AFNOR U 44-041 (1985). Cette norme ne mentionne que la teneur maximale en élément total ( $200 \mathrm{mg} \mathrm{Se} \cdot \mathrm{kg}^{-1}$ matière sèche) et ne prend donc pas en compte les facteurs de biodisponibilité et de toxicité, intimement liés aux types d'association de l'élément avec les différents constituants des boues et des sols et à la forme physicochimique sous laquelle il est présent. En effet, le sélénium peut exister sous forme minérale, aux degrés d'oxydation $-I I, 0,+I V$ et $+\mathrm{VI}$, ou sous forme organique (acides aminés séléniés ou composés méthylés volatils) au degré d'oxydation - II. Chacune de ces formes est plus ou moins disponible pour les plantes et les animaux et toxique à des seuils plus ou moins bas ( $D L_{50}$ de Se(IV): $3,5 \mathrm{mg} \mathrm{Se} \cdot \mathrm{kg}^{-1}$ (WHO, 1987) et $\mathrm{DL}_{50}$ du diméthylséléniure : $1600 \mathrm{mg} \mathrm{Se} \cdot \mathrm{kg}^{-1}$ (AL BAYATl et al., 1992)). II est donc indispensable de mesurer le sélénium total dans un échantillon, mais également d'en réaliser la spéciation. Nous avons choisi dans cette étude de séparer le sélénium en fractions plus ou moins disponibles (spéciation de phases), puis de réaliser sur chacune d'elle la détermination des différentes formes physico-chimiques sous lesquelles le sélénium est présent (spéciation d'espèces). Les moyens analytiques nécessaires, qui n'existent que depuis peu d'années, permettent maintenant d'aborder ces problèmes. De plus, l'utilisation de deux techniques, la Spectrométrie d'Absorption Atomique ElectroThermique (ETAAS) et la Voltamétrie de Redissolution Cathodique Différentielle Pulsée (DPCSV), ainsi que d'échantillons certifiés permet de confirmer la fiabilité de nos résultats.

\section{MATÉRIEL ET MÉTHODES}

\section{Échantillons}

\section{Boues de station d'épuration française}

La station d'épuration d'eaux résiduaires de Tarbes Ouest a été choisie comme site d'étude en raison de sa proximité et de sa représentativité. Cette station, d'une capacité de $\mathbf{4 0} 000$ équivalents habitants, fonctionne en aération prolongée avec un niveau de rejet NK1 (soit moins de $50 \mathrm{mg} \mathrm{N} \cdot \mathrm{L}^{-1}$ pour un échantillon moyen de deux heures ou moins de $40 \mathrm{mg} \mathrm{N} \cdot \mathrm{L}^{-1}$ pour un échantillon moyen de vingt-quatre heures) (DEGREMONT, 1989). La ligne d'eau est composée d'un prétraitement usuel, d'un bassin d'aération type chenal avec aérovis, suivis d'un clarificateur avant rejet. Les boues sont déshydratées sur filtre à bande avant d'être valorisées en agriculture. Les échantillons utilisés pour cette étude proviennent des boues destinées à l'épandage. Ils ont été autoclavés à $120^{\circ} \mathrm{C}$ pendant $20 \mathrm{~min}$, étape indispensable pour pouvoir les conserver suffisamment longtemps pour la mise au point des techniques analytiques tout en étant sûr que l'évolution bactérienne ne viendra pas fausser les résultats. Il est à noter que ce type de traitement n'intervient pas sur la répartition des espèces séléniées étudiées. Une partie des échantillons a ensuite été conservée telle quelle (humidité résiduelle $85,4 \%)$ à $-20^{\circ} \mathrm{C}$ alors que la deuxième moitié était lyophilisée pendant $120 \mathrm{~h}$ (humidité résiduelle $2,7 \%$ ) avant d'être également stockée à $-20^{\circ} \mathrm{C}$. 


\section{Matériaux certifiés}

Les échantillons certifiés ont été obtenus auprès du BCR (Bruxelles, Belgique). Dans la mesure où aucun matériau certifié ne correspondait exactement à la boue naturelle étudiée (boue domestique de faible concentration en sélénium), nous en avons sélectionné deux : la boue CRM 145 R (essentiellement d'origine industrielle - concentration indicative : $3,3 \mathrm{mg} \mathrm{Se} \cdot \mathrm{kg}^{-1}$ poids sec) et la boue CRM 007 (représentative d'une zone résidentielle américaine avec une influence industrielle - valeur de certification : $15,0 \pm 1,8 \mathrm{mg} \mathrm{Se} \cdot \mathrm{kg}^{-1}$ poids sec).

\section{Réactifs}

Toutes les solutions sont préparées à partir d'eau suprapure (résistance $18 \mathrm{M} \Omega$ ) obtenue par le système MilliRO/MilliQ (Millipore).

Les solutions mères à $1000 \mathrm{mg} \mathrm{Se} \cdot \mathrm{L}^{-1}$ de $\mathrm{Se}(\mathrm{IV})$ et $\mathrm{Se}(\mathrm{VI})$ sont préparées respectivement à partir de séléniure de sodium $\left(\mathrm{Na}_{2} \mathrm{SeO}_{3}, 5 \mathrm{H}_{2} \mathrm{O}\right)(\mathrm{MERCK}$, pro analysis) et d'acide sélénique $\left(\mathrm{H}_{2} \mathrm{SeO}_{4} 96 \%\right.$ ) (MERCK, pro analysis). Les acides aminés, séléno-DL-méthionine et séléno-DL-cystine, ont été obtenus chez SIGMA. Toutes les solutions pour les ajouts dosés et les droites de calibration sont préparées quotidiennement par dilutions successives de cette solution mère.

Les réactifs utilisés pour les extractions $(\mathrm{NaOH})$, les minéralisations $\left(\mathrm{HNO}_{3}\right.$ $65 \%, \mathrm{H}_{2} \mathrm{O}_{2} 30 \%$ ), les réductions $(\mathrm{HCl} 37 \%)$, l'élution des espèces fixées sur la résine ( $\mathrm{NaCl})$ et l'électrolyte support $\left(\mathrm{H}_{2} \mathrm{SO}_{4} 96 \%\right)$ sont tous des produits suprapurs (MERCK).

La solution tampon de phosphate d'ammonium-acide citrique est préparée dans $100 \mathrm{~mL}$ à partir de $2,1 \mathrm{~g}$ d'acide citrique, $1,15 \mathrm{~g}$ de phosphate mono-ammonique et $5 \mathrm{~mL}$ d'éthanol $(5 \%(\mathrm{v} / \mathrm{v}))$ amenés à $\mathrm{pH}=7,5$ par de l'ammoniaque concentré. Tous les produits utilisés proviennent de PROLABO RP Normapur.

Le modificateur de matrice utilisé en ETAAS est obtenu par dilution au dixième d'une solution mère de palladium (II) à $5,3 \mathrm{mg} \mathrm{Pd} \cdot \mathrm{mL}^{-1}$, préparée par dissolution de $\left(\mathrm{NO}_{3}\right)_{2} \mathrm{Pd}, 2 \mathrm{H}_{2} \mathrm{O}$ de chez SIGMA dans $\mathrm{HNO}_{3} 1 \%$.

L'Amberlite CG-400 (sous forme R-Cl, résine de type I, 100-200 mesh) en provenance de Prolabo a été utilisée pour la spéciation d'espèces.

\section{Appareillage}

Les mesures par DPCSV sont réalisées sur un appareillage PAR 264A, équipé d'une électrode à goutte de mercure pendante PAR 303A, d'une électrode de référence $\mathrm{Ag} / \mathrm{AgCl}$ saturée en $\mathrm{KCl}$, d'une électrode auxiliaire en platine et d'une table traçante Kipp and Zonen $X-Y$ recorder.

Les mesures par ETAAS sont réalisées grâce à un spectromètre Unicam 939 (four Unicam GF 90) couplé à un passeur d'échantillon FS 90. Les données sont traitées par le logiciel Solaar AA System. Toutes les mesures ont été faites avec une super lampe à cathode creuse, une correction de fond par effet Zeeman et l'utilisation d'un modificateur de matrice au palladium.

Les conditions analytiques utilisées ont été précédemment optimisées pour ces échantillons (HENINGER et al., 1996a). 
Les minéralisations assistées par micro-ondes focalisées sont réalisées sur un Microdigest 301 (système à pression atmosphérique) de PROLABO équipé du programmateur TX32, d'une pompe à trois voies pour l'ajout des réactifs et du système Aspivap. Des matras en Téflon et en quartz ont été utilisés pour les minéralisations mais des matras en verre borosilicaté sont suffisants pour les étapes de réduction.

Toute la verrerie est décontaminée par séjour dans $\mathrm{HNO}_{3} 10 \%$ pendant $48 \mathrm{~h}$ et triple rinçage à l'eau suprapure.

\section{Méthodes}

Tous les protocoles de prétraitement des échantillons ont été appliqués à 5 prises d'essai.

\section{Extraction}

Les extractions sont réalisées sur 0,25 à $2 \mathrm{~g}$ de boue (suivant l'échantillon), placés dans un tube à hémolyse en présence de $10 \mathrm{~mL}$ de l'extractant choisi et soumis à sonication pendant $3 \mathrm{~h}$. L'extrait est séparé du résidu solide par centrifugation à $4000 \mathrm{tr} \cdot \mathrm{min}^{-1}$ pendant $30 \mathrm{~min}$. L'extrait est alors minéralisé pour l'analyse du sélénium total (puis éventuellement réduit pour les analyses par DPCSV, méthode spécifique de Se(IV)) ou analysé directement par DPCSV pour la détermination de $\mathrm{Se}(\mathrm{IV})$.

\section{Minéralisation}

La minéralisation permet de solubiliser les formes séléniées par destruction de la matrice et de transformer les formes organiques en formes minérales (majoritairement $\mathrm{Se}(\mathrm{VI})$ ). La mise au point des protocoles de minéralisation sur plaque à induction (HENINGER et al., 1996a) ou par micro-ondes focalisées (HENINGER et al., 1996b) a été précédemment décrite.

\section{Réduction}

La DPCSV étant une technique spécifique de Se(IV), il est nécessaire de transformer le $\mathrm{Se}(\mathrm{VI})$ obtenu lors de la minéralisation. Une étape de réduction à chaud par $\mathrm{HCl} 6 \mathrm{M}$ doit donc être effectuée avant l'analyse. Nous avons utilisé le protocole de SEBY (1994) pour la réduction classique et adapté celui de MUNOZOLIVAS (1996) pour la réduction assistée par micro-ondes.

\section{Schéma de spéciation du sélénium}

La figure 1 résume les différentes étapes qui ont conduit à la détermination du sélénium total et à sa spéciation dans les boues.

L'analyse après minéralisation, et éventuellement réduction, des surnageants et culots obtenus lors des étapes d'extraction permet d'obtenir le pourcentage de sélénium extrait, mais également de réaliser un bilan en sélénium à comparer avec la concentration en sélénium total obtenue précédemment afin d'évaluer les éventuelles pertes en sélénium ayant pu se produire lors des manipulations.

Une analyse directe de l'extrait (surnageant) par DPCSV permet de déterminer la teneur en Se(IV) de l'échantillon. 


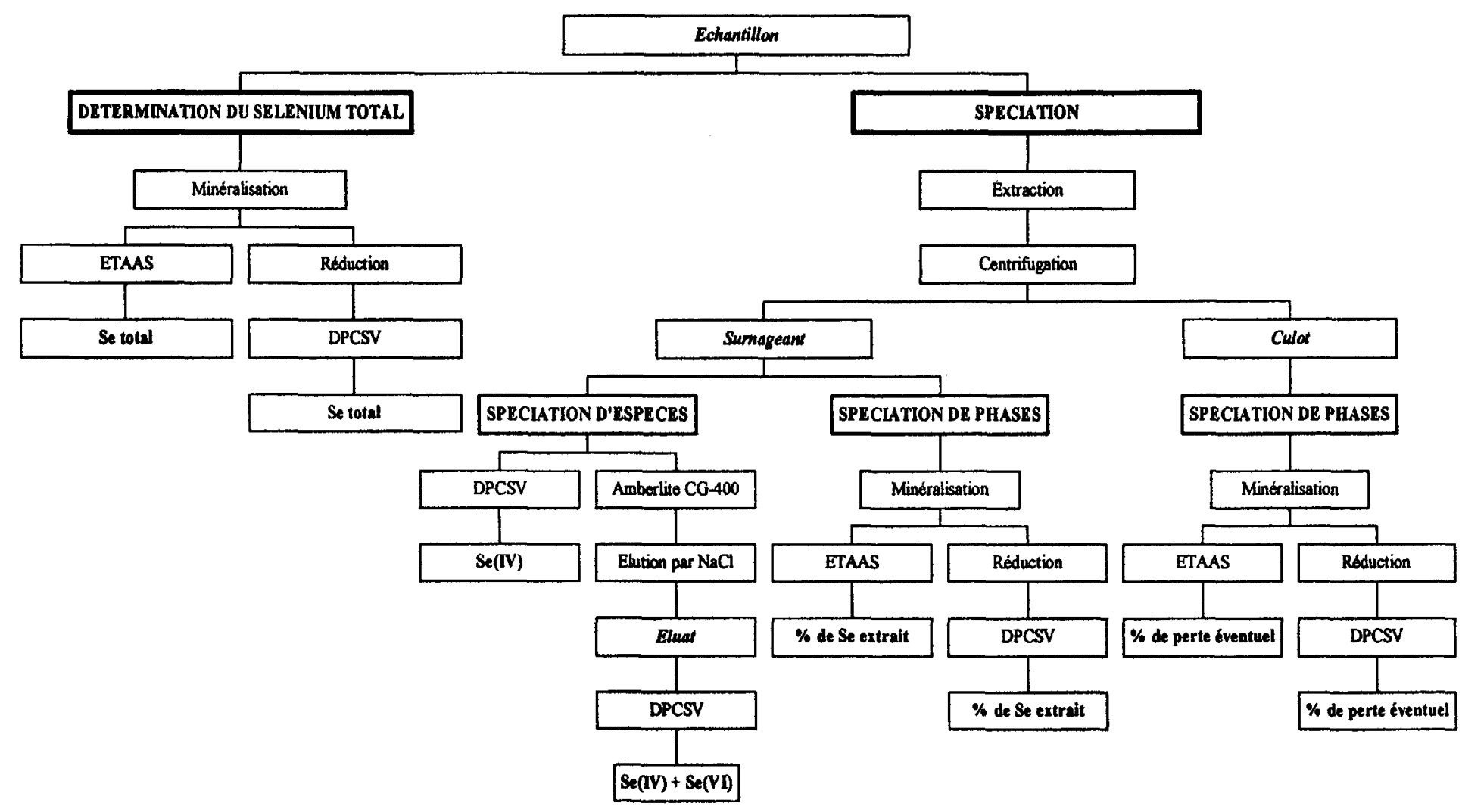

Figure 1 Schéma de spéciation du sélénium dans les boues de stations d'épuration d'eaux résiduaires urbaines.

Speciation scheme for selenium in sewage sludge samples. 
L'utilisation d'une résine échangeuse d'anions (Amberlite CG-400 (R-CI)) permet de séparer les formes organiques des formes inorganiques, par fixation sélective à $\mathrm{pH}=4$ de ces dernières. On peut ainsi récupérer par élution au $\mathrm{NaCl}$ concentré la somme Se(IV) + Se(VI) et réaliser un dosage de cette fraction après réduction sans observer d'interférence provenant de la fraction organique.

\section{RÉSULTATS ET DISCUSSION}

\section{Dosage du sélénium total}

Des études préalables sur les échantillons certifiés ont montré que le mélange $\mathrm{HNO}_{3} / \mathrm{H}_{2} \mathrm{O}_{2} / \mathrm{H}_{2} \mathrm{O}$ permettait d'obtenir les meilleurs résultats lors des minéralisations, tout en minimisant les problèmes d'interférence pour les deux techniques analytiques choisies (HENINGER et al., 1996a). L'utilisation des micro-ondes a permis d'obtenir une réduction des temps de minéralisation de un jour à une heure (HENINGER et al., 1996b). La réduction a également été amenée d'une durée de 45 min à seulement 2 min.

Les résultats moyens obtenus par les deux techniques analytiques (sans distinction du type de minéralisation et de réduction) sont regroupés dans le tableau 1.

Tableau 1 Résultats en sélénium total obtenus pour les échantillons de boues de station d'épuration certifiés et les échantillons de la station d'épuration d'eaux résiduaires de Tarbes.

Table 1 Total Se concentrations obtained for sewage sludge certified samples and for the sewage sludge from Tarbes.

\begin{tabular}{|c|c|c|c|c|}
\hline \multirow[t]{2}{*}{ Échantillon } & \multicolumn{2}{|c|}{$\begin{array}{l}\text { Concentration moyenne } \\
\text { (mg Se } \cdot \mathrm{kg}^{-1} \text { poids sec) }\end{array}$} & \multicolumn{2}{|c|}{$\begin{array}{l}\text { Ecart-type relatif } \\
\text { RSD }(\%)\end{array}$} \\
\hline & DPCSV & ETAAS & DPCSV & ETAAS \\
\hline $\begin{array}{l}\text { CRM } 007 \\
\left(15,0 \pm 1,8 \mathrm{mg} \mathrm{Se} \cdot \mathrm{kg}^{-1} \text { poids sec }\right)\end{array}$ & $15,28 \pm 1,20$ & $15,23 \pm 1,5$ & 8 & 10 \\
\hline $\begin{array}{l}\text { CRM 145R } \\
\left(3,3 \mathrm{mg} \mathrm{Se} \cdot \mathrm{kg}^{-1} \text { poids sec) }\right.\end{array}$ & $3,45 \pm 0,35$ & $3,30 \pm 0,30$ & 10 & 9 \\
\hline Boue d'épandage lyophilisée & $1,08 \pm 0,11$ & $1,12 \pm 0,03$ & 10 & 3 \\
\hline Boue d'épandage humide & $1,05 \pm 0,19$ & $1,06 \pm 0,14$ & 18 & 13 \\
\hline
\end{tabular}

Les valeurs correspondant aux échantillons du BCR sont en bon accord avec les valeurs certifiées, validant ainsi nos protocoles analytiques.

Les écarts types relatifs (RSD) obtenus sur les échantillons lyophilisés ( $3 \%$ $10 \%$ ) attestent de la répétabilité satisfaisante de ces traitements. Les valeurs plus fortes obtenues pour les échantillons humides sont probablement liées à une mauvaise homogénéisation du matériau. 


\section{Spéciation de phases}

Pour réaliser la spéciation d'un élément il est nécessaire de l'extraire de son milieu, tout en assurant une préservation de la répartition initiale des espèces et en évitant les pertes.

Cette spéciation de phases implique l'utilisation d'un certain nombre de réactifs différents. Les extractions séquentielles ayant été critiquées par GRUEBEL et al. (1988) pour leur manque de sélectivité et l'accumulation d'erreurs qu'elles entraînent, nous avons choisi de travailler sur une extraction parallèle, c'est-à-dire d'utiliser les réactifs séparément sur des prises d'essai distinctes.

Dans tous les cas les mécanismes impliqués lors de l'utilisation des extractants doivent correspondre aux réactions physico-chimiques ou biologiques rencontrées dans le milieu naturel, afin de pouvoir associer chaque surnageant obtenu à une ou plusieurs fractions de sélénium. Des différentes fractions considérées par les auteurs dans la littérature (CHAO et SANZOLONE (1989), STERRITT et LESTER (1980), ELLIOTT et al. (1981)), il est possible d'en dégager quatre principales : la fraction soluble (espèces solubles et sélénium non spécifiquement adsorbé), la fraction échangeable (sélénium spécifiquement adsorbé qui sera relargué par échange d'ions), la fraction dite "oxydable " (sélénium associé aux oxydes de $\mathrm{Fe}, \mathrm{Al}$ et $\mathrm{Mn}$, aux carbonates et à la matière organique hydrolysable) et la fraction dite " minérale " (sélénium inclus dans la structure cristalline du milieu). La somme des fractions soluble et échangeable représente le sélénium directement disponible pour les plantes s'il est transféré dans les sols par le biais des eaux de ruissellement. II faudra bien évidemment tenir compte ensuite des affinités de ces plantes pour les différentes formes présentes. La fraction dite " oxydable " représente le sélénium potentiellement disponible par le biais d'une mobilisation chimique ou microbienne. En effet, une modification des conditions redox du milieu aurait pour effet de permettre le relargage du sélénium associé aux oxydes de $\mathrm{Fe}, \mathrm{Al}$ et $\mathrm{Mn}$, aux carbonates ou à la matière organique hydrolysable, sous une forme soluble et donc disponible (CHAO et SANZOLONE, 1989).

II existe en fait peu d'articles concernant l'extraction du sélénium des boues de stations d'épuration (STERRITT et LESTER (1980), ELLIOTT et al. (1981)). Cette étude s'inspire donc de travaux concernant l'extraction du sélénium des sols (SEBY (1994), CHAO et SANZOLONE (1989), KANG et al. (1991), KANG et al. (1993), YAMADA et HATTORI (1990), GUSTAFSSON et JOHNSSON (1992), WANG et SIPPOLA (1990)), ou de cendres (NISS et al., 1993). Nous avons ainsi choisi trois types d'extractants: l'eau chaude pour la fraction soluble, un mélange phosphate d'ammonium - acide citrique pour la somme des fractions soluble et échangeable, et la soude $2 \mathrm{M}$ pour la somme des fractions soluble, échangeable et " oxydable". La somme des quatre fractions est obtenue par minéralisation de l'échantillon. Par soustraction du résultat obtenu pour l'extractant précédent (réactif plus faible) il est aisé d'évaluer le pourcentage de chaque fraction.

Ces différents extractants ont été préalablement testés sur la boue CRM 007 afin d'optimiser les techniques analytiques pour les différents milieux, les extraits par la soude entraînant en particulier des projections dans le four de l'ETAAS (HENINGER et al., 1996a).

Les résultats obtenus pour l'échantillon certifié CRM 007 sont reportés sur la figure 2.

Il est à noter que nous n'avons trouvé aucune étude dans la littérature pouvant servir de référence pour le contrôle de ces résultats. 


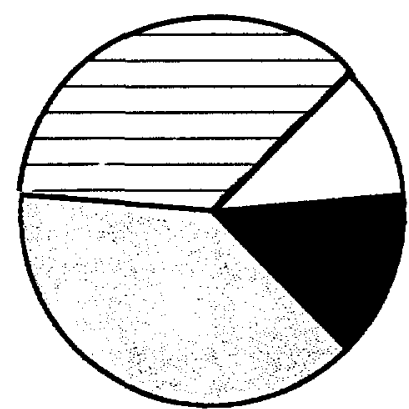

DFraction soluble (11\%)

- Fraction échangeable (14\%)

口Fraction "oxydable" (39\%)

日Fraction "minérale" (36\%)

Figure 2 Répartition du sélénium contenu dans CRM 007.

Selenium speciation in CRM 007.

Le même type de protocole a été appliqué à la boue de Tarbes sous forme lyophilisée (figure 3) ainsi qu'à la forme humide (boue déshydratée brute) (figure 4).

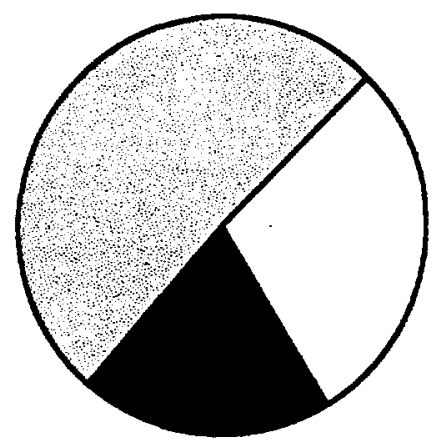

口Fraction soluble (29\%)

Fraction échangeable (20\%)

Fraction "oxydable" (51\%)

Figure 3 Répartition du sélénium dans la boue de Tarbes lyophilisée.

Selenium speciation in the freeze-dried sewage sludge from Tarbes.

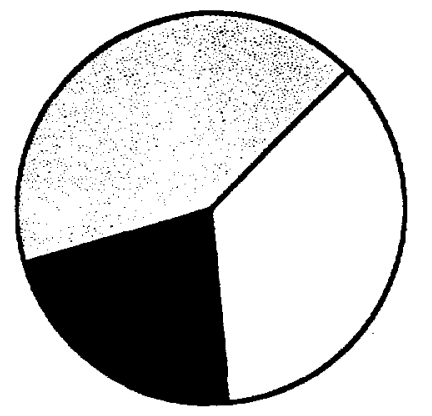

$\square$ Fraction soluble (36\%)

Fraction échangeable (22\%)

$\square$ Fraction "oxydable" (42\%)

Figure 4 Répartition du sélénium dans la boue de Tarbes humide.

Selenium speciation in the wet sewage sludge from Tarbes. 
II semblerait que le sélénium soit beaucoup plus disponible dans la boue de Tarbes que dans l'échantillon certifié. Ceci peut s'expliquer par la nature exclusivement urbaine de cet échantillon. Le sélénium y est probablement présent sous une forme plus soluble et la matrice moins réfractaire que dans les boues d'origine même partiellement industrielle. Une étude partielle réalisée sur la boue CRM 145 R (boue d'origine industrielle) confirme cette tendance puisque seulement $1 \%$ du sélénium total est présent dans cet échantillon sous forme soluble. II n'existe à notre connaissance aucune donnée dans la littérature permettant de corroborer ces résultats où d'en fournir une explication précise.

La boue de Tarbes sous forme humide subit une extraction plus importante par les milieux aqueux que la boue lyophilisée. Ceci peut être mis en relation directe avec l'humidité résiduelle du matériau. En effet, le volume de surnageant était toujours légèrement supérieur dans le cas de la boue humide par récupération lors de la centrifugation de l'eau interstitielle. Dans le matériau humide il s'est probablement produit un équilibre entre le sélénium en phase aqueuse et le sélénium en phase particulaire et lors de l'extraction le sélénium déjà solubilisé a pu être également récupéré, ce qui peut s'avérer plus délicat avec la boue sous forme lyophilisée.

Il est également important de noter que tout le sélénium présent a pu être extrait par la soude $2 \mathrm{M}$ comme le montrent les résultats du tableau 2.

Tableau 2 Résultat des extractions à la soude sur les échantillons de Tarbes.

Table 2 Results concerning $\mathrm{NaOH}$ extractions of $\mathrm{Se}$ in the sludge samples from Tarbes.

\begin{tabular}{|c|c|c|c|c|c|}
\hline \multirow{2}{*}{ Échantillons } & \multirow{2}{*}{$\begin{array}{l}\text { Prise d'essai } \\
\text { (g) }\end{array}$} & \multicolumn{2}{|c|}{$\begin{array}{l}\text { Concentration moyenne } \\
\text { (mg Se } \cdot \mathrm{kg}^{-1} \text { poids sec) }\end{array}$} & \multicolumn{2}{|c|}{$\begin{array}{c}\text { Écart-type relatif } \\
\text { RSD (\%) }\end{array}$} \\
\hline & & DPCSV & ETAAS & $\begin{array}{l}\text { DPCSV } \\
(n=5)\end{array}$ & $\begin{array}{l}\text { ETAAS } \\
(n=5)\end{array}$ \\
\hline \multicolumn{6}{|l|}{ BOUE LYOPHILISÉE } \\
\hline - Se total & $0,25-1$ & $1,08 \pm 0,11$ & $1,12 \pm 0,03$ & 10 & 3 \\
\hline - Se extrait par $\mathrm{NaOH} 2 \mathrm{M}$ & 1 & $1,07 \pm 0,03$ & $1,20 \pm 0,07$ & 3 & 6 \\
\hline \multicolumn{6}{|l|}{ BOUE HUMIDE } \\
\hline - Se total & $1-2$ & $1,05 \pm 0,19$ & $1,06 \pm 0,14$ & 18 & 13 \\
\hline - Se extrait par $\mathrm{NaOH} 2 \mathrm{M}$ & 2 & $1,03 \pm 0,17$ & $4,29 \pm 0,41$ & 16 & 10 \\
\hline
\end{tabular}

En ce qui concerne les analyses par DPCSV on observe une excellente corrélation entre les valeurs obtenues pour le sélénium total et les valeurs obtenues dans les extraits basiques. Par ETAAS les résultats ne sont pas encore très satisfaisants. Pour les extraits de boue lyophilisée la valeur moyenne un peu forte obtenue par cette technique peut être justifiée par les fonds assez importants observés lors de l'analyse $(\approx 0,8 \mathrm{DO})$, dûs au sodium apporté par la soude. Dans le cas de la boue sous forme humide on observe une exaltation du signal dont l'origine n'a pas pu être encore clairement identifiée. Il semble qu'elle soit liée à la présence d'interférants, tels que le fer (HOENIG, 1991), libérés par ce processus d'extraction appliqué à un échantillon humide. Des études sont actuellement en cours pour tenter d'expliquer ce phénomène. 
Ces résultats prouvent par ailleurs que la totalité du sélénium présent dans la boue de Tarbes est potentiellement disponible. Plus de la moitié du sélénium présent (58 \%) est par ailleurs directement disponible par lessivage. Quoiqu'il en soit toutes les espèces séléniées n'étant pas aussi aisément assimilables par les plantes il est intéressant d'examiner également la répartition des espèces.

\section{Spéciation d'espèces}

Une analyse directe des extraits basiques par DPCSV permet de déterminer la teneur en $\mathrm{Se}(\mathrm{IV})$. Néanmoins cette analyse nécessite un passage en milieu acide qui entraîne la précipitation des acides humiques présents dans l'échantillon (POTIN-GAUTIER et al., 1995), ce qui perturbe les mesures. II est à noter que le milieu est trop colloĩdal pour que l'on puisse séparer de façon efficace la phase soluble de la phase particulaire afin de déterminer quel pourcentage de sélénium est inclus dans le précipité. Néanmoins, des études réalisées au laboratoire sur des extraits de sols ont montré qu'il était assez faible (POTIN-GAUTIER et al.). Les teneurs en Se(IV) de la boue de Tarbes ont donc pu être estimées, en tenant compte de ce paramètre, à $30-40 \%$ du sélénium total.

Des études préliminaires ont par ailleurs démontré que les acides aminés séléniés (sélénométhionine et sélénocystine) sont totalement transformés lors de l'étape de réduction nécessaire à la détermination de Se(VI) par DPCSV (tableau 3).

Tableau 3 Influence de l'étape de réduction sur la stabilité des acides aminés séléniés.

Table 3 Influence of the reduction step on seleno-amino acid compounds.

\begin{tabular}{|c|c|c|c|c|c|c|c|c|}
\hline \multicolumn{3}{|c|}{ Composition de la solution } & \multicolumn{5}{|c|}{ Concentration en Se ( $\mu \mathrm{g} \mathrm{Se} . \mathrm{I}^{-1}$ ) } & \multirow{2}{*}{$\begin{array}{l}\text { Pourcentage de } \\
\text { transformation } \\
\text { du sélénium orga } \\
\text { nique en Se(IV) } \\
\text { après réduction }\end{array}$} \\
\hline Espèces & $\mu \mathrm{g} \mathrm{Se} \cdot \mathrm{r}^{-1}$ & $\begin{array}{c}\text { DPCSV } \\
\text { (analyse } \\
\text { directe) }\end{array}$ & $\begin{array}{l}\text { RSD } \\
(\%)\end{array}$ & $\begin{array}{l}\text { DPCSV } \\
\text { (après } \\
\text { réduction) }\end{array}$ & $\begin{array}{l}\text { RSD } \\
(\%)\end{array}$ & $\begin{array}{l}\text { ETAAS } \\
\text { (Se total) }\end{array}$ & $\begin{array}{l}\text { RSD } \\
(\%)\end{array}$ & \\
\hline SeM & 20 & n.d. & - & $20 \pm 2$ & 12 & $20,8 \pm 0,4$ & 1 & 100 \\
\hline $\mathrm{SeC}$ & 20 & n.d. & - & $20,4 \pm 0,6$ & 3 & $21,6 \pm 0,4$ & 2 & 100 \\
\hline $\operatorname{Se}(I V)+\operatorname{SeM}$ & $10+10$ & $10,4 \pm 0,4$ & 4 & $21 \pm 2$ & 9 & $20,8 \pm 0,2$ & 1 & 100 \\
\hline $\mathrm{Se}(\mathrm{IV})+\mathrm{SeC}$ & $10+10$ & $9,6 \pm 0,7$ & 7 & $21 \pm 1$ & 5 & $20,2 \pm 0,4$ & $<1$ & 100 \\
\hline $\operatorname{Se}(V I)+\operatorname{SeM}$ & $10+10$ & n.d. & - & $21,1 \pm 0,7$ & 3 & $21,8 \pm 0,3$ & 1 & 100 \\
\hline $\operatorname{Se}(V I)+\operatorname{SeM}$ & $20+10$ & n.d. & - & $30 \pm 2$ & 7 & $31 \pm 1$ & 3 & 100 \\
\hline
\end{tabular}

n.d. : non détecté.

SeM : sélénaméthionine.

$\mathrm{SeC}$ : sélénocystine.

II est donc nécessaire d'utiliser une résine pour séparer les formes inorganiques des formes organiques. Cette séparation étant optimale pour un $\mathrm{pH}$ de 4 (HENINGER, 1994), on observe là encore un phénomène de précipitation des acides humiques. La résine a donc été utilisée en réacteur agité afin d'éviter les phénomènes de colmatage et, en tenant compte des incertitudes liées à la précipitation des acides humiques, nous avons pu déterminer un taux de $\mathrm{Se}$ (VI) dans les échantillons de boue de la station d'épuration de Tarbes de 2 à $20 \%$. 
Ces résultats préliminaires sont intéressants puisqu'ils permettent d'avoir une première indication sur les teneurs en $\mathrm{Se}(\mathrm{IV})$ et $\mathrm{Se}(\mathrm{VI})$ (formes les plus toxiques et facilement assimilables par les plantes) présentes dans ces matrices. Néanmoins, le développement de nouvelles méthodes de caractérisation semble préférable pour une évaluation plus précise des teneurs rencontrées.

\section{CONCLUSION}

Nous avons mis au point des protocoles analytiques permettant la détermination du sélénium total dans des boues de stations d'épuration d'eaux résiduaires. La détermination du sélénium total n'étant pas suffisante pour juger de sa toxicité nous avons également mis au point un schéma d'extraction parallèles permettant de déterminer le sélénium réellement et potentiellement disponible lors d'un épandage sur sol agricole. Bien que les teneurs mesurées dans les échantillons de boues de la station d'épuration de Tarbes soient encore très largement inférieures à la norme NF U 44-041, la totalité du sélénium présent est potentiellement disponible pour l'environnement. Trente à quarante pour-cent de cet élément sont par ailleurs présents sous forme de Se(IV) et zéro à vingt pour-cent sous forme de $\mathrm{Se}(\mathrm{VI})$. Le développement de techniques de couplages de plus en plus précises et sensibles, telles que la Chromatographie Liquide Haute Performance - Plasma d'Argon lonisé - Spectrométrie de Masse (HPLC-ICP-MS), devrait à terme permettre de réaliser cette spéciation d'espèces de façon plus efficiente.

\section{REMERCIEMENTS}

Les auteurs souhaitent remercier MM. Guillot et Vermont, responsables de la station d'épuration de Tarbes, et tous les membres du CIRSEE pour leur aide lors de cette étude, ainsi que la Lyonnaise des Eaux-Dumez pour le financement de ces travaux.

\section{RÉFÉRENCES BIBLIOGRAPHIQUES}

AL BAYATI M.A., RAABE O.G., TEAGUE S.V., 1992. Effect of inhalated dimethyl selenide in the fisher 344 male rat. J. Toxicol. Environ. Health, 37, 549-557.
CAMBIER P., 1994. Contamination of soils by heavy metals and other trace elements : a chemical perspective. Analusis, 22(2), 2124. 
CHAO T.T., SANZOLONE R.F., 1989. Fractionation of soil selenium by sequential partial dissolution. Soil Sci. Soc. Am. J., 53, 385-392.

DEGREMONT, 1989. Mémento technique de l'eau, Tome 1, $9^{e}$ édition, 586-587.

DUBOIS F., BELLEVILLE F., 1988. Sélénium : rôle physiologique et intérêt en pathologie humaine. Pathol. Biol., 1017-1025.

ELLIOTT G.E.P., MARSHALL B.W., SMITH A.C., 1981. Aspects of the analysis of selenium in water treatment wastes. Anal. Proc., 18(2), 64-67.

GRUEBEL K.A., DAVIS J.A., LECKIE J.O., 1988. The feasibility of using sequential extraction techniques for arsenic and selenium in soils and sediments. Soil. Sci. Soc. Am. J., 52, 390-398.

GUSTAFSSON J.P., JOHNSSON L., 1992. Selenium retention in the organic matter of Swedish forest soils. J. Soil Sci., 43, 461-472.

HENINGER, 1994. Spéciation du sélénium dans les eaux après préconcentration sur résine. Étude des conditions de stockage. Rapport de D.E.A., 24 p.

HENINGER I., POTIN-GAUTIER M., ASTRUC M., GALVEZ L., VIGNIER V., 1996b. Influence des micro-ondes sur la minéralisation des boues de stations d'épuration pour la détermination du sélénium. Journées Information Eaux, Poitiers, France, 19-20 septembre, 10p.

HENINGER I., POTIN-GAUTIER M., ASTRUC M., SNIDARO D., VIGNIER V., MANEM J., 1996a. Selenium in sewage sludge. General aspects and analytical challenge. Intern. J. Environ. Anal. Chem., sous presse.

HOENIG M., 1991. Détermination du sélénium dans le sang par spectrométrie d'absorption atomique electrothermique avec effet Zeeman, discussion des paramètres critiques. Analusis, 19, 41-46.

KANG Y., YAMADA H., KYUMA K., HATTORI T., 1993. Speciation of selenium in soil. Soil Sci. Plant Nutr., 39(2), 331-337.

KANG Y., YAMADA H., KYUMA K., 1991. Selenium in soil humic acid. Soil Sci. Plant Nutr., 37(2), 241-248.

MUNOZ-OLIVAS R., 1996. Spéciation du sélénium par techniques de couplage et détection par ICP/MS : application à la production des matériaux de référence. Thèse d'Université $N^{\circ} 1482$, Université de Bordeaux I, Laboratoire de Photophysique et Photochimie Moléculaire, 351 Cours de la Libération, F33405 Talence, France, $272 \mathrm{p}$.

NF U 44-041, 1985. Matières fertilisantes Boues des ouvrages de traitement des eaux usées urbaines, Association Française de Normalisation, Tour Europe, cedex 7, 82080 Paris-la-Défense, France, $11 \mathrm{p}$.

NISS N.D., SCHABRON J.F., BROWN T.H., 1993. Determination of selenium species in coal fly ash extracts. Environ. Sci. Technol., 27(5), 827-829.

PERRIN D., IMPENS R., 1995. L'utilisation agricole des boues de station d'épuration urbaine en Europe. Tribune de l'Eau, 576(4), 31-34.

POTIN-GAUTIER M., SEBY F., ASTRUC M., 1995. Interference of humic substances on the speciation analysis of inorganic selenium in waters and soils by DPCSV. Fresenius Journal of Analytical Chemistry, 351, 443-448.

SEBY F., 1994. Optimisation de méthode analytiques pour la spéciation du sélénium inorganique dans les eaux et les sols. Thèse d'Université $N^{\circ} 235$, Université de Pau et des Pays de l'Adour, Laboratoire de Chimie Analytique, Avenue de l'Université, F64000 Pau, France, $154 \mathrm{p}$.

STERRITT R.M., LESTER J.N., 1980. The value of sewage sludge to agriculture and effects of the agricultural use of sludges contaminated with toxic elements: a review. Sci. Tot. Environ., 16, 55-90.

VALIRON F., 1994. Mémento du gestionnaire de l'alimentation en eau et de l'assainissement. éd. Lavoisier Tech. \& Doc., Tome 2, 569-579.

WANG D., SIPPOLA J., 1990. Selenium in soils extracts and plants determined by fluorometry. Annales Agriculturae Fenniae, 29, 151-156.

WORLD HEALTH ORGANIZATION (WHO), 1987. Environmental Health Criteria 58 : Selenium. Genève, Suisse, $306 \mathrm{p}$.

YAMADA H., HATTORI T., 1990. Determination of soluble selenium in soils. Soil Sci. Plant Nutr., 36(1), 163-166. 